\title{
Repeat associated mechanisms of genome evolution and function revealed by the Mus caroli and Mus pahari genomes
}

David Thybert, ${ }^{1,2}$ Maša Roller, ${ }^{1}$ Fábio C.P. Navarro, ${ }^{3}$ Ian Fiddes, ${ }^{4}$ Ian Streeter, ${ }^{1}$ Christine Feig, ${ }^{5}$ David Martin-Galvez, ${ }^{1}$ Mikhail Kolmogorov, ${ }^{6}$ Václav Janoušek, ${ }^{7}$ Wasiu Akanni, ${ }^{1}$ Bronwen Aken, ${ }^{1}$ Sarah Aldridge, ${ }^{5,8}$ Varshith Chakrapani, ${ }^{1}$ William Chow, ${ }^{8}$ Laura Clarke, ${ }^{1}$ Carla Cummins, ${ }^{1}$ Anthony Doran, ${ }^{8}$ Matthew Dunn, ${ }^{8}$ Leo Goodstadt, ${ }^{9}$ Kerstin Howe, ${ }^{3}$ Matthew Howell, ${ }^{1}$ Ambre-Aurore Josselin, ${ }^{1}$ Robert C. Karn, ${ }^{10}$ Christina M. Laukaitis, ${ }^{10}$ Lilue Jingtao, ${ }^{8}$ Fergal Martin, ${ }^{1}$ Matthieu Muffato, ${ }^{1}$ Stefanie Nachtweide, ${ }^{11}$ Michael A. Quail, ${ }^{8}$ Cristina Sisu, ${ }^{3}$ Mario Stanke, ${ }^{11}$ Klara Stefflova, ${ }^{5}$ Cock Van Oosterhout, ${ }^{12}$ Frederic Veyrunes, ${ }^{13}$ Ben Ward, ${ }^{2}$ Fengtang Yang, ${ }^{8}$ Golbahar Yazdanifar, ${ }^{10}$ Amonida Zadissa, ${ }^{1}$ David J. Adams, ${ }^{8}$ Alvis Brazma, ${ }^{1}$ Mark Gerstein, ${ }^{3}$ Benedict Paten, ${ }^{4}$ Son Pham, ${ }^{14}$ Thomas M. Keane, ${ }^{1,8}$ Duncan T. Odom, ${ }^{5,8}$ and Paul Flicek ${ }^{1,8}$

${ }^{1}$ European Molecular Biology Laboratory, European Bioinformatics Institute, Wellcome Genome Campus, Hinxton, Cambridge CB10 1SD, United Kingdom; ${ }^{2}$ Earlham Institute, Norwich Research Park, Norwich NR4 7UH, United Kingdom; ${ }^{3}$ Yale University Medical School, Computational Biology and Bioinformatics Program, New Haven, Connecticut 06520, USA; ${ }^{4}$ Department of Biomolecular Engineering, University of California, Santa Cruz, California 95064, USA; ${ }^{5}$ University of Cambridge, Cancer Research UK Cambridge Institute, Robinson Way, Cambridge CB2 ORE, United Kingdom; ${ }^{6}$ Department of Computer Science and Engineering, University of California, San Diego, La Jolla, California 92092, USA; ${ }^{7}$ Department of Zoology, Faculty of Science, Charles University in Prague, 12844 Prague, Czech Republic; ${ }^{8}$ Wellcome Sanger Institute, Wellcome Genome Campus, Hinxton, Cambridge, CB10 1 SA, United Kingdom; ${ }^{9}$ Wellcome Trust Centre for Human Genetics, Oxford OX3 7BN, United Kingdom; ${ }^{10}$ Department of Medicine, College of Medicine, University of Arizona, Tuscon, Arizona 85724, USA; ${ }^{11}$ Institute of Mathematics and Computer Science, University of Greifswald, Greifswald 17487, Germany; ${ }^{12}$ School of Environmental Sciences, University of East Anglia, Norwich Research Park, Norwich NR4 7TJ, United Kingdom; ${ }^{13}$ Institut des Sciences de I'Evolution de Montpellier, Université Montpellier/CNRS, 34095 Montpellier, France; ${ }^{14}$ Bioturing Inc, San Diego, California 92121, USA

\begin{abstract}
Understanding the mechanisms driving lineage-specific evolution in both primates and rodents has been hindered by the lack of sister clades with a similar phylogenetic structure having high-quality genome assemblies. Here, we have created chromosome-level assemblies of the Mus caroli and Mus pahari genomes. Together with the Mus musculus and Rattus norvegicus genomes, this set of rodent genomes is similar in divergence times to the Hominidae (human-chimpanzee-gorilla-orangu$\tan$ ). By comparing the evolutionary dynamics between the Muridae and Hominidae, we identified punctate events of chromosome reshuffling that shaped the ancestral karyotype of Mus musculus and Mus caroli between 3 and 6 million yr ago, but that are absent in the Hominidae. Hominidae show between four- and sevenfold lower rates of nucleotide change and feature turnover in both neutral and functional sequences, suggesting an underlying coherence to the Muridae acceleration. Our system of matched, high-quality genome assemblies revealed how specific classes of repeats can play lineage-specific roles in related species. Recent LINE activity has remodeled protein-coding loci to a greater extent across the Muridae than the Hominidae, with functional consequences at the species level such as reproductive isolation. Furthermore, we charted a Muridae-specific retrotransposon expansion at unprecedented resolution, revealing how a single nucleotide mutation transformed a specific SINE element into an active CTCF binding site carrier specifically in Mus caroli, which resulted in thousands of novel, species-specific CTCF binding sites. Our results show that the comparison of matched phylogenetic sets of genomes will be an increasingly powerful strategy for understanding mammalian biology.
\end{abstract}

[Supplemental material is available for this article.]

\footnotetext{
Corresponding authors: duncan.odom@cruk.cam.ac.uk, flicek@ebi. ac.uk

Article published online before print. Article, supplemental material, and publication date are at http://www.genome.org/cgi/doi/10.1101/gr.234096.117. Freely available online through the Genome Research Open Access option.
}

(C) 2018 Thybert et al. This article, published in Genome Research, is available under a Creative Commons License (Attribution 4.0 International), as described at http://creativecommons.org/licenses/by/4.0/. 
One of the justifications for sequencing many mammalian genomes is to compare these with each other to gain insight into core mammalian functions and map lineage-specific biology. For example, the discovery of human accelerated regions, including the HAR1 gene linked to brain development, relied on comparison between the human and chimpanzee genomes (Pollard et al. 2006). Across the mammalian clade, the choice of species to be sequenced and their relative priority have been based on a combination of factors including their value as model organisms (Mouse Genome Sequencing Consortium 2002; Rat Genome Sequencing Project Consortium 2004; Lindblad-Toh et al. 2005) or agriculture species (The Bovine Genome Sequencing and Analysis Consortium 2009; Groenen et al. 2012) as well as the value for comparative genome analysis (Lindblad-Toh et al. 2005, 2011). Despite the extreme popularity of mouse and rat as mammalian models, there have been few efforts to sequence the genomes of other closely related rodent species, although greater understanding of their specific biology would almost certainly enhance their value as models.

Comparing genome sequences identifies both novel and conserved loci likely to be responsible for core biological functions (Lindblad-Toh et al. 2005), phenotypic differences (Atanur et al. 2013; Liu et al. 2014; Foote et al. 2015), and many other lineagespecific characteristics (Kim et al. 2011; Wu et al. 2014; Foote et al. 2015). Indeed, evolutionary comparisons have even enabled the identification of genomic variation, such as repeat expansions, which can explain aspects of genome and karyotype evolution (Carbone et al. 2014).

Even closely related species can exhibit large-scale structural changes ranging from lineage-specific retrotransposon insertions to karyotype differences. The mechanisms driving these changes may vary between mammalian lineages, and the reasons for these differences remain mostly unknown. For example, the rate of chromosomal rearrangement in mammals can vary dramatically between lineages: Murid rodents have a rate that has been estimated to be between three times and hundreds of times faster than in primates (Murphy et al. 2005; Capilla et al. 2016). Transposable elements and segmental duplications have often been found enriched in the vicinity of chromosomal breakpoints (Bailey et al. 2004; The Bovine Genome Sequencing and Analysis Consortium 2009; Carbone et al. 2014). It is not clear whether these transposable elements directly cause chromosomal rearrangement by triggering nonallelic homologous recombination (NAHR) (Janoušek et al. 2013) or if they indirectly act via factors such as chromatin structure or epigenetic features (Capilla et al. 2016).

Transposable elements typically make up $40 \%$ of a mammalian genome, have variable activity across lineages, and thus can evolutionarily and functionally shape genome structure (Kirkness et al. 2003; Ray et al. 2007). Retrotransposons have numerous links to novel lineage-specific function (Kunarso et al. 2010; Irie et al. 2016). For instance, pregnancy in placental mammals may have been shaped by an increase of activity of the MER20 retrotransposon, which has rewired the gene regulatory network of the endometrium (Lynch et al. 2011). Furthermore, Alu elements have expanded several times in primates with the largest event occurring around 55 million yr ago (MYA) (Batzer and Deininger 2002), while SINE B2 elements widely expanded in murid rodents (Kass et al. 1997). Retrotransposons can affect gene expression by altering pre-mRNA splicing (Lin et al. 2008) or regulatory networks (Jacques et al. 2013; Chuong et al. 2016). For example, lineage-specific transposons can carry binding sites for regulators including the repressor NRSF/REST (Mortazavi et al. 2006; Johnson et al. 2007) and CTCF (Bourque et al. 2008; Schmidt et al. 2012).
The rate of fixation of single nucleotide mutation can also change between different mammalian lineages, for example rodents have a faster rate than primates (Mouse Genome Sequencing Consortium 2002). One likely explanation is the shorter generation time observed in rodents compared to primates ( $\mathrm{Li}$ and Tanimura 1987; Li et al. 1996). In this hypothesis, most single nucleotide mutations occur during DNA replication in the male germline, and the larger number of passages associated with the rodent's shorter generation time accumulates more mutations in the same period of time (Goetting-Minesky and Makova 2006).

Thus far, the dynamics of genome evolution between mammalian lineages have been mainly studied by comparing distant genomes (Rat Genome Sequencing Project Consortium 2004; Murphy et al. 2005; The Bovine Genome Sequencing and Analysis Consortium 2009; Lindblad-Toh et al. 2011; Foote et al. 2015), and less frequently using closely related species (Carbone et al. 2014; Capilla et al. 2016). Comparing distantly related species can lead to poor resolution of genome structural changes and an inability to assess mechanisms or initial drivers of change. This is due in part to incomplete or uncertain alignments between distant genomes and the inability to unravel multiple evolutionary events that may have occurred in a single genomic region.

At present, primates are one of the mammalian clades (if not the only one) with enough sequenced genomes (The Chimpanzee Sequencing and Analysis Consortium 2005; Rhesus Macaque Genome Sequencing and Analysis Consortium 2007; Locke et al. 2011; Scally et al. 2012; Carbone et al. 2014; Gordon et al. 2016) to facilitate high-resolution studies of genome evolution within a single mammalian lineage (Marques-Bonet et al. 2009; Gazave et al. 2011; Schwalie et al. 2013; Navarro and Galante 2015). It remains uncertain whether the evolutionary dynamics observed in the primates are common across other mammalian clades.

In this study, we generated high-quality genome assemblies for both Mus caroli and Mus pahari to create a sister clade for comparison with primate genome evolution. The combination of the Mus caroli and Mus pahari genomes with the reference mouse and rat genomes mirror, in divergence time and phylogenetic structure, the four Hominidae species with sequenced genomes (human, chimp, gorilla, orangutan). Here, we directly compare the processes of genome sequence evolution active within Hominidae and Muridae as two representative clades of mammals.

\section{Results}

Sequencing, assembly, and annotation of Mus caroli and Mus pahari genomes

We sequenced the genomes of Mus caroli and Mus pahari females using a strategy combining overlapping Illumina paired-end and long mate-pair libraries with OpGen optical maps (Supplemental Fig. S1A; Supplemental Methods SM1.1-SM1.4). First, scaffolds were created with ALLPATHS-LG (Gnerre et al. 2011) from the overlapping and 3-kb Illumina mate-pair libraries and then were coupled to the OpGen optical maps to yield 3079 (Mus caroli) and 2944 (Mus pahari) super scaffolds with a N50 of 4.3 and 3.6 $\mathrm{Mb}$, respectively. We reconstructed pseudochromosomes by guiding the assembly based on (1) chromosome painting information and (2) multiple, closely related genomes, effectively reducing the assembly bias caused by using only a single reference genome (Kolmogorov et al. 2016). We obtained 20 and 24 chromosomes with a total assembled genome size of 2.55 and $2.47 \mathrm{~Gb}$, respectively, for Mus caroli and Mus pahari. These two genomes have 
assembly statistics comparable to the available primate genomes, including chimpanzee, gorilla, and orangutan (Supplemental Fig. S1B).

We generated RNA-seq data from brain, liver, heart, and kidney in Mus caroli and Mus pahari to annotate the genes using an integration of TransMap (Stanke et al. 2008), AUGUSTUS (Stanke et al. 2006), and AUGUSTUS-CGP (Konig et al. 2016) pipelines (Supplemental Methods SM1.7). This approach identified 20,323 and 20,029 protein-coding genes and 10,069 and 9336 noncoding genes, comparable to the mouse and rat reference genomes (Supplemental Fig. S2A).

The assembled Mus caroli and Mus pahari genomes have a low nucleotide error rate, estimated as one sequencing error every 25 $30 \mathrm{~kb}$ based on mapping the mate-pair libraries back to the final corresponding genome assemblies (Supplemental Fig. S1C; Supplemental Methods SM1.14). Comparison of the optical maps with the final genome assemblies suggests that up to 3035 and 1691 genomic segments could be misassembled, representing $2.5 \%$ and $3.1 \%$ of the Mus caroli and Mus pahari genomes, respectively (Supplemental Fig. S1D). To estimate the gene completion of the two assemblies, we inspected the alignment coverage of protein-coding genes conserved across all vertebrates (Supplemental Methods SM1.15). The alignment coverage was 93.3\% and 93.2\% for the Mus caroli and Mus pahari assemblies, respectively, values that fall within the range (91.6\%-94.7\%) for corresponding primate genomes (Supplemental Fig. S2B).

Previous phylogenetic analyses of the Mus genus have relied on the sequence of cytochrome b, 12S rRNA, and the nuclear Irbp gene to broadly estimate a 2.9-7.6 MY divergence among the Mus caroli, Mus pahari, and Mus musculus species (Veyrunes et al. 2005; Chevret et al. 2014). We refined this estimate using the whole-genome assemblies to create a complete collection of the fourfold degenerate sites found in amino acids conserved across mammals. In specific and highly conserved amino acids, the third base within the coding triplet is thought to be under virtually no selective constraint, meaning neutral rates of change can be estimated by comparing the accumulation of mutations within these sites. We then estimated the divergence time separating Mus musculus with Mus caroli and Mus pahari by anchoring our analysis on a mouse-rat divergence time of $12.5 \mathrm{MY}$, an estimate based on fossil records (Supplemental Fig. S3A; Supplemental Methods SM1.16; Fig. 1A; Jacobs and Flynn 2005).

Our estimates show that Mus pahari diverged from the Mus musculus lineage 6 MYA with a 95\% confidence interval ranging from 5.1 to 7.5 MY, and Mus caroli diverged 3.0 MYA with a 95\% confidence interval ranging from 2.6 to $3.8 \mathrm{MY}$ (Fig. 1A). We observed no introgression or incomplete lineage sorting among these four species that could affect the divergence time estimate (Supplemental Fig. S3B; Supplemental Methods SM1.17). These results were robust to (1) the choice of the gene categories from which we selected the fourfold degenerate sites and (2) the evolutionary model used to make the divergence estimates (Supplemental Fig. S3A; Supplemental Methods SM1.16).

\section{A punctuated event of chromosomal rearrangements shaped the Mus musculus and Mus caroli ancestral karyotype}

In rodents, chromosome numbers evolve much more rapidly than among most other mammalian clades including primates (Ferguson-Smith and Trifonov 2007). To compare the evolutionary dynamics of large ( $>3 \mathrm{Mb})$ inter-chromosomal rearrangements, we performed pairwise whole-genome alignments of the Muridae and Hominidae genomes (Fig. 1B; Supplemental Fig. S4). Hominidae karyotypes, like most mammalian clades, are highly stable, typically showing only one or two unique breaks for each species (Fig. 1C; Ferguson-Smith and Trifonov 2007).

In contrast, our analysis revealed that the Muridae clade appears to have been subjected to punctate periods of accelerated genome instability interspersed with periods of more typical stability. For example, a period of massive genome rearrangement occurred in the shared ancestor of Mus caroli and Mus musculus after the split with Mus pahari (3-6 MYA) that resulted in 20 synteny breaks found only in Mus caroli and Mus musculus (Fig. 1C, D). Notably, over the most recent 0-3 MY, the karyotypes of Mus caroli and Mus musculus have been stable with no large genome rearrangements. Second, rat shows 19 lineage-specific synteny breaks when compared with Mus pahari, but it counts substantially more (35 synteny breaks) when compared to Mus musculus or Mus caroli. This means that the rat karyotype more closely resembles that of Mus pahari than the karyotypes of the two other Mus species. Regardless of whether the rat-specific changes were introduced gradually or in one or more punctuated events, the overall impact on the genome (approximately 20 large breaks) is vastly greater than observed in Hominidae in a roughly corresponding divergence time (orangutan versus human: 1 large break) (Fig. 1C; Supplemental Fig. S4).

In order to find a potential molecular mechanism driving the punctate increases of inter-chromosomal rearrangement, we asked if the inter-chromosomal breakpoints between Mus musculus and Mus pahari were enriched in repeat elements. Repeat elements are thought to drive chromosome rearrangement by increasing local homology and then inducing NAHR (Hedges and Deininger 2007; Robberecht et al. 2013). We found a significant enrichment of LTR retrotransposons with a concurrent age of the rearrangement events, i.e., 3-6 MY old (empirical $P$-value, $P<10^{-3}$ ) (Supplemental Fig. S5). We also found an enrichment, although not statistically significant, of SINE elements of the same age. When considering the set of repeats of all ages, there was no observed enrichment at breakpoints for any type of repeat (Supplemental Fig. S5). This result is compatible with a model in which specific LTR repeats increase local susceptibility to interchromosomal rearrangement by NAHR. However, our analysis does not rule out that LTR integration and inter-chromosomal rearrangement could co-occur in the same location without a causal relationship. Indeed, local genomic properties, such as chromatin structure are known hot spots for both transposable element integration and chromosomal breakpoints (Capilla et al. 2016; Sultana et al. 2017).

In summary, our results detail a punctate event of chromosome reshuffling that happened in the Muridae lineage between 3 and 6 MYA and that has led to the observed karyotype of laboratory mice. Furthermore, our analysis revealed an association of 3to 6-million-year-old LTR elements at the chromosomal breakpoints, suggesting a potential connection between this class of retrotransposons and the mechanisms driving these large-scale events in rodents.

Divergence and turnover of genomic sequences and segments are accelerated in Muridae, particularly for LINE retrotransposons

We next tested whether the genome of Muridae evolves faster than that of Hominidae by comparing the rate of nucleotide variation within each clade. We focused on the whole genome (Supplemental Fig. S6; Supplemental Methods SM3.1; Fig. 2) and

\section{Genome Research}

www.genome.org 


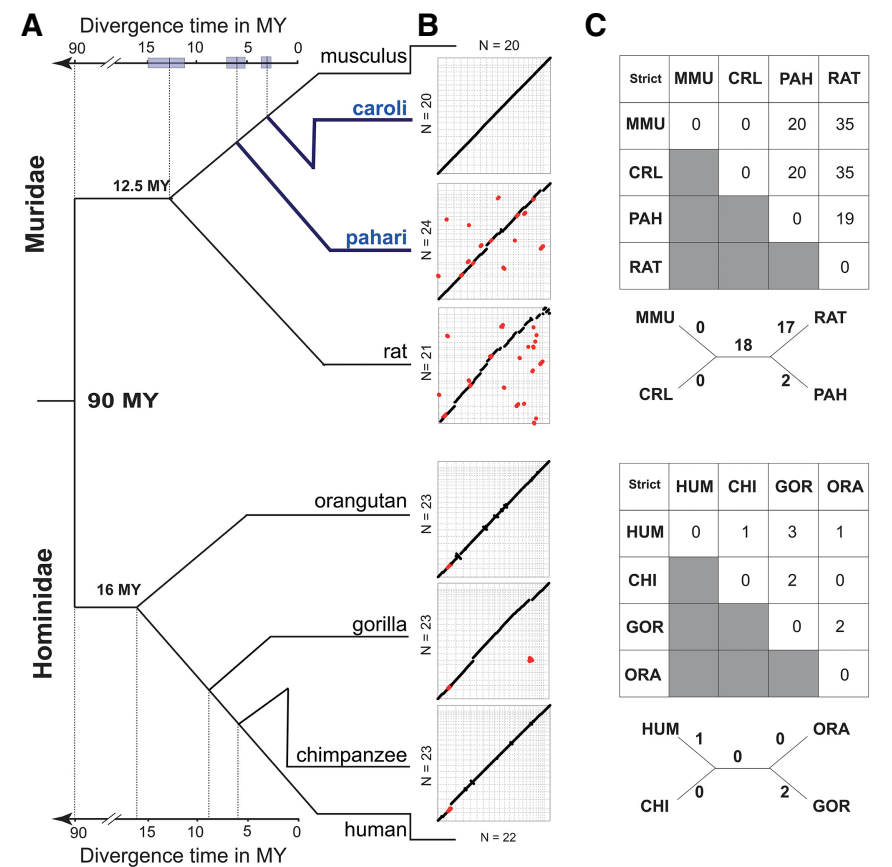

D
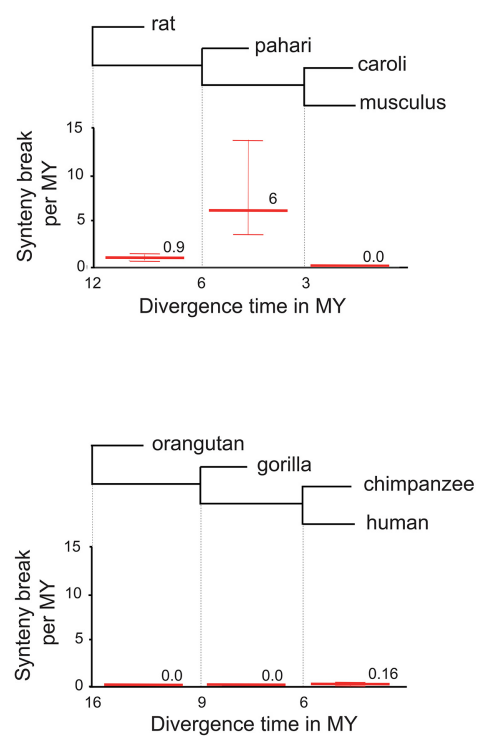

Figure 1. Muridae genomes undergo large chromosomal rearrangements in punctuate bursts, resulting in greater structural diversity than primates. $(A)$ Phylogenetic tree showing that the divergence time of the four Muridae species mirrors that of the four Hominidae species. The Mus species in blue were sequenced and assembled for this study. The $95 \%$ confidence interval of the divergence time estimation is shown by the shaded boxes (Supplemental Methods SM1.16). (B) Dot plots of whole-genome pairwise comparison between Mus musculus and the three other Muridae (top), and between human and the three other Hominidae (bottom). The chromosomes of Mus musculus and human were ordered by chromosome number. The chromosomes of the other species were ordered to optimize the contiguity across the diagonal. Red dots represent large ( $>3 \mathrm{Mb}$ ) inter-chromosomal rearrangements (fusion/ fission and translocation). (C) Matrix of neighbor-joining tree of synteny breaks involving inter-chromosomal rearrangement for Muridae and Hominidae: (MMU) Mus musculus; (CAR) Mus caroli; (PAH) Mus pahari; (RAT) rat; (HUM) Human; (CHI) chimpanzee; (GOR) gorilla; (ORA) orangutan. (D) The rate of synteny breaks between sequential internal branch points of the Muridae and Hominidae clades. Muridae have undergone a punctuate increase in the rate of syntenic breaks between 3 and 6 MYA.

found that the Muridae clade shows a sixfold increase in the rate of change when compared to the Hominidae clade.

We took a similar approach to establish how rapidly sequence changes occur in the whole genome as well as in specific classes of genomic elements, including ancestral repeats such as LTR, SINE, LINE, and DNA repeats, exons, and CTCF binding motifs (Fig. 2). The rate of nucleotide variation change reflects different evolutionary constraints, consistent with Gaffney and Keightley (2006) (Fig. 2A). Nevertheless, across all inspected categories, Muridae genome evolution is accelerated between six- and sevenfold when compared to primates (Fig. 2C).

We next quantified how rapidly entire genomic segments are gained and lost among these four rodent species. Similar to nucleotide variation, different types of elements show differing rates of turnover (Fig. 2B). Because DNA transposons, as opposed to retrotransposons, lost their activity early in the primate and rodent lineages (Mouse Genome Sequencing Consortium 2002; Pace and Feschotte 2007), we used the empirically observed turnover of DNA transposons as a background rate. Notably, this background rate of DNA repeat evolution in rodents is approximately 4.5fold higher than in Hominidae.

In both clades, protein-coding exons are more stable than DNA transposons, as expected. In contrast, both SINE and LTR retrotransposons are actively expanding in a lineage-specific manner and show higher turnover than DNA transposons in both rodents and primates. (Fig. 2B,C). Moreover, in both clades, the rates of SINE and LTR element turnover are similar to each other and, when compared to the turnover rate of DNA transposons, exhibit approximately the same relative increase. This suggests that Muridae and Hominidae have a generally comparable activity of SINE and LTR retrotransposons when compared to DNA transposons. However, in Muridae, LINE retrotransposons are roughly 1.5 times more active than LTR and SINE elements and appear to have greatly accelerated activity when compared to the rate found in primates (ANCOVA, $P$-value $<10^{-3}$ ) (Fig. 2B,C). This result is consistent with previous reports showing increased lineage-specific LINE activity in mouse as compared to human (Mouse Genome Sequencing Consortium 2002).

In summary, our results detail the remarkably rapid evolution of Muridae genomes. Common classes of repeat elements expand between 4.1- and 7.7-fold faster in rodents than in Hominidae genomes. Most notably, LINE retrotransposon activity is highly accelerated in Muridae and has typically resulted in the birth of several hundred megabases of novel genomic sequence (69-374 $\mathrm{Mb}$ ) in each assayed rodent genome.

\section{Accelerated LINE retrotransposon activity has shaped coding gene evolution in rodents}

We next asked how retrotransposon activity has changed during the evolutionary history of both clades. We first estimated in each genome the age of every retrotransposon by calculating the sequence identity between the retrotransposon and the consensus sequence, which is an approximation of the ancestral repeat. 
A
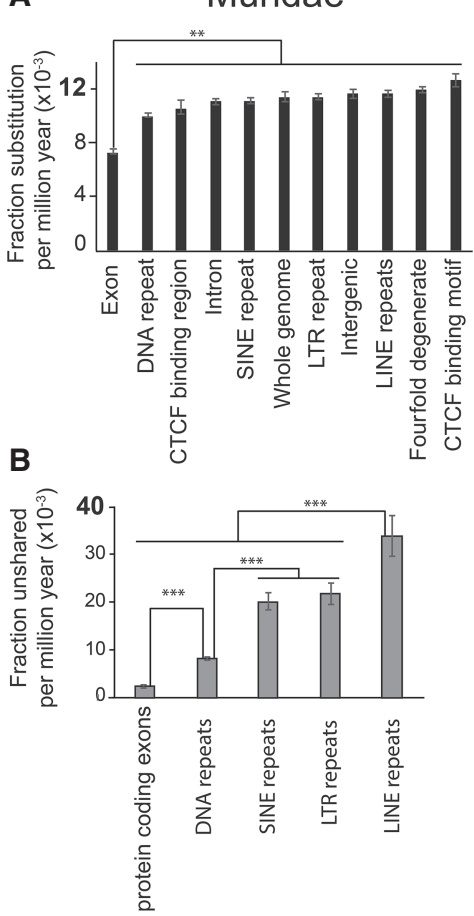

C

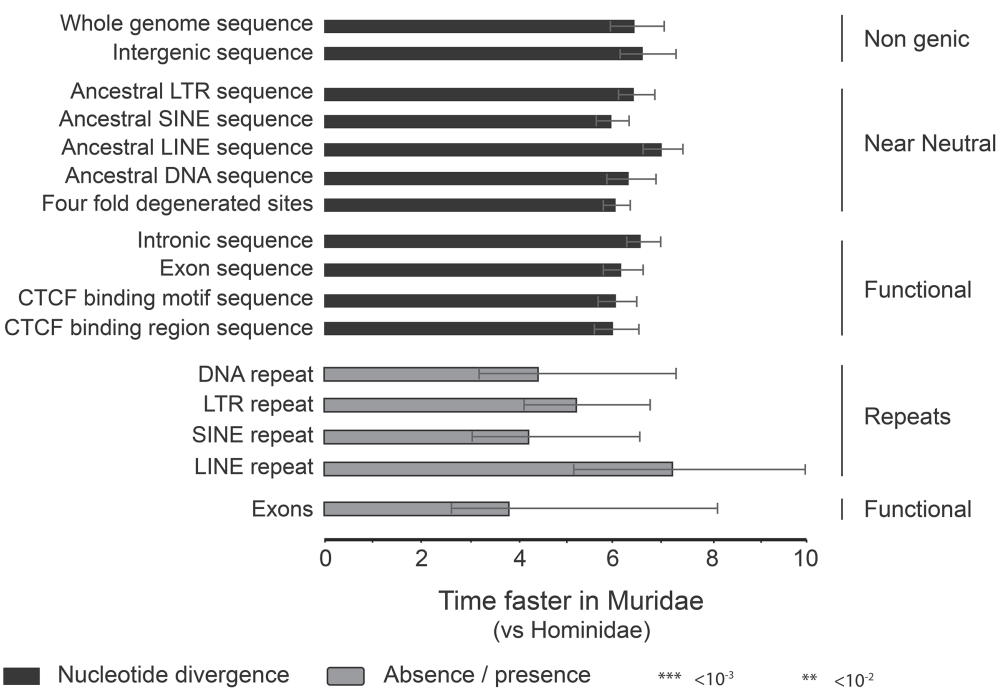

Figure 2. Acceleration of mutational rates in the Muridae lineage. (A) The evolutionary rate of nucleotide variation calculated for specific genomic regions. The error bar represents the standard error within the $95 \%$ confidence interval. (B) The rate of segmental turnover calculated for specific genomic regions. The error bar represents the standard error within the 95\% confidence interval (Supplemental Methods SM3.2). (C) The bar chart shows the ratios of evolutionary rates between Muridae and Hominidae. Mouse versus human ratios were calculated for rates of nucleotide divergence (black bars) and the turnover rates (gray bars) for specific genomic regions (Supplemental Methods SM3.2).

Because the sequence of transposable elements evolves nearly neutrally, the relationship between the sequence identity and the estimated age of a repeat is approximately linear (Supplemental Methods SM4.1; Liu et al. 2009).

Our analysis confirmed previous reports (Batzer and Deininger 2002) that a major event of SINE Alu element retrotransposition occurred in the primate lineage, peaking at 55 MYA and subsequently decreasing to the current basal activity (Fig. 3A). In contrast, LINE and LTR elements show relatively low but consistent activity during primate evolution, whereas DNA transposons show essentially no activity (Fig. 3A). As in primates, LTR elements in rodents also appear to be relatively quiescent over recent evolutionary time. For SINE elements in the Muridae, there has been a consistent level of moderate activity including insertion events from the SINE B2 family previously shown to carry a CTCF binding site (Bourque et al. 2008; Schmidt et al. 2012).

The most striking difference in retrotransposition activity between the Hominidae and Muridae clades is the greatly accelerated expansion of LINE elements in rodents beginning 8.5 MYA, which has continued at an elevated activity level (Fig. 3A). This increase has resulted in a substantial enrichment $(6 \%-$ 14\%; Fisher's exact test, $P<10^{-16}$ ) of species-specific LINE retrotransposons in all four Muridae species (Supplemental Fig. S7).

The LINE-L1 retrotranscriptase machinery can reshape mammalian genomes by capturing RNAs and reinserting retrotranscribed copies into the genome, as in the case for processed pseudogenes (Esnault et al. 2000). We observed an increase of the number of retrocopies with an age matching the evolutionary window as the recent LINE expansion in rodents (Fig. 3B). This increase of 9-million-yr-old retrocopies was not found in Hominidae genomes, which instead show a peak of $\sim 50$-million-yr-old retrocopies. We also found a small number of chimeric transcripts caused by retrogene insertions in Muridae genomes (Supplemental Fig. S8; Supplemental Methods SM4.4).

In addition, LINE retrotransposons can act as substrate for NAHR, thus driving segmental duplication and leading to copy number variation and gene cluster expansion (Startek et al. 2015; Janoušek et al. 2016). The secretoglobin $(S c g b)$ gene cluster containing $S c g b 1 b$ and $S c g b 2 b$ genes, also called the androgen binding protein $(A b p)$ gene cluster containing Abpa and $A b p b g$ genes (Laukaitis et al. 2008), illustrates this effect. $A b p$ is involved in mating preference (Laukaitis and Karn 2012) and incipient reinforcement in the hybrid zone where the geographic range of two mouse subspecies make secondary contact (Bimova et al. 2011). Since the mouse-rat ancestor, this gene cluster has progressively expanded in the Muridae lineage with the greatest number of copies observed in the Mus musculus genome (Fig. 3C). Importantly, in the four genomes, LINE retrotransposons are enriched within the $A b p$ gene cluster compared either with

\section{Genome Research}

www.genome.org 
A
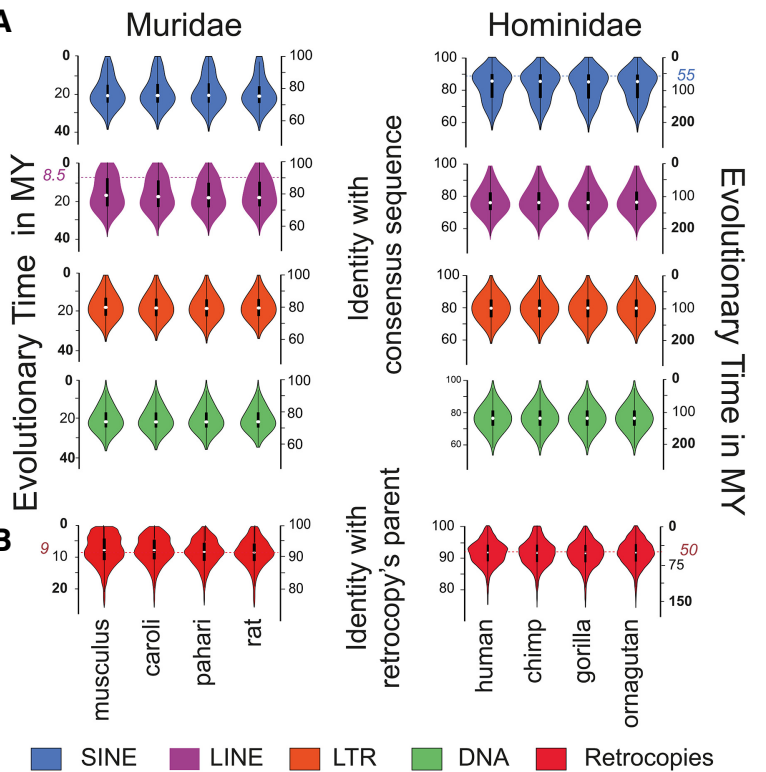

C

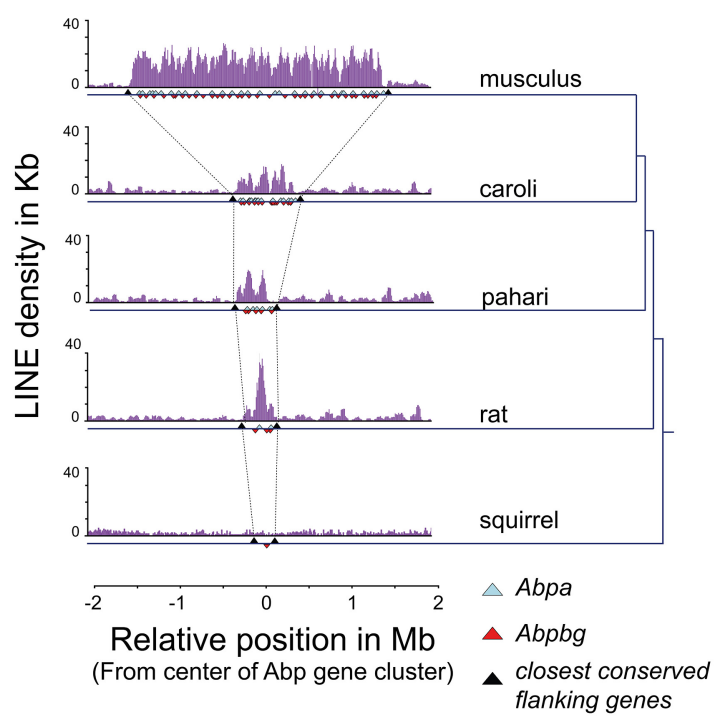

Figure 3. Recent LINE activity can remodel protein-coding gene loci. (A) Violin plots showing the distribution of repeat elements that have the indicated divergence from the ancestral element sequence: (blue) SINE; (purple) LINE; (orange) LTR; (green) DNA. The age of the transposable elements was estimated using the nucleotide divergence from ancestral SINE, LINE, LTR, and DNA elements (Supplemental Methods SM4.1). The dashed lines indicate the estimated peaks of the most recent expansions in Mus musculus and human. (B) Violin plots showing the distribution of retrocopies (red) that have the indicated divergence from their parental genes for each Muridae (left) and Hominidae (right) species. The age of the retrocopies was estimated by the nucleotide divergence from ancestral retrocopies and the corresponding parental genes (Supplemental Methods SM4.3). The dashed line indicates the peak of the most recent expansion in Mus musculus. (C) Representation of the density of LINE elements in the Abp gene cluster for Mus musculus, Mus caroli, Mus pahari, the rat, and the thirteen-lined ground squirrel. The blue and red triangles represent the Abp genes: (blue) Abpa $(S c g b 1 b)$; (red) Abpbg (Scgb2b). The black triangles represent the closest flanking genes (upstream [Scn1b] and downstream [Gpi1]) shared by the four Muridae species and the squirrel.

adjacent intergenic regions (empirical $P$-value, $P<10^{-5}$ ) or with collections of single genes matched for total gene number (empirical $P$-value, $P<10^{-2}$ ) (Supplemental Methods SM4.6; Fig. 3C). In comparison, no LINE enrichment was observed in the 13-lined ground squirrel (Ictidomys tridecemlineatus) genome, where only one copy of $A b p$ gene is present (Fig. 3C). LTR elements are also enriched within the $A b p$ gene cluster in the Muridae genomes (empirical $P$-value, $P<10^{-5}$ ) (Supplemental Fig. S9).

Taken together, the dramatic, recent, and still-active expansion of LINE activity in rodents has had important functional consequences for the Muridae genome, ranging from a wave of retrocopy integrations to gene cluster expansions.

\section{Retrotransposition of SINE B2_Mm1 elements drove a species-specific expansion of CTCF occupancy in Mus caroli}

Previous studies have shown that the SINE B2 element carries a CTCF binding motif and can thus drive the expansion of CTCF binding in rodents (Bourque et al. 2008; Schmidt et al. 2012). We took advantage of the closely related Muridae genomes to investigate the molecular mechanisms behind this expansion. We determined the genome-wide binding for CTCF in livers of the four Muridae by performing ChIP-seq experiments (Fig. 4A; Supplemental Methods SM1.11). In addition, we used a previously published data set to identify CTCF genome-wide binding in immortalized lymphoblast cells from four primate species (Schwalie et al. 2013). We found between $\sim 24,000$ and 48,000 CTCF binding sites across the four Muridae species and between $\sim 21,000$ and 57,000 across the four Hominidae species (Supplemental Fig. S10A).
As expected, the CTCF binding sites were overrepresented in SINE retrotransposons in Muridae compared to Hominidae (Fisher's exact test, $P$-val $<10^{-6}$ ) (Fig. 4B; Supplemental Fig. S10B). SINE elements carrying a CTCF binding site were enriched in SINE B2 compared to random expectation (empirical $P$-value, $P<10^{-5}$ ) (Supplemental Fig. S10C). We then asked if any particular mouse species showed enhanced $\mathrm{B} 2$ retrotransposition resulting in novel lineage-specific CTCF binding sites. We estimated the age of the $\mathrm{B} 2$ elements in the four Muridae species and found an overrepresentation of young elements positive for CTCF binding in Mus caroli (Fig. 4C). Based on the distribution of repeat ages, this recent wave of CTCF binding site expansion started early in the Mus caroli lineage 3 MYA. In comparison, the Hominidae genomes show no similar expansion of CTCF occupancy driven by retrotransposition (Supplemental Fig. S11).

Next, we asked whether the Mus caroli-specific expansion of CTCF binding could be attributed to a particular SINE B2 subfamily. We found an overrepresentation of SINE B2_Mm1 occupied by CTCF specifically in Mus caroli when compared with the other rodents (empirical $P$-value, $P<10^{-5}$ ) (Supplemental Fig. S10D). Among the 20,248 B2_Mm1 elements in Mus caroli, 16\% (4151) showed CTCF binding in vivo. In contrast, a significantly smaller fraction of B2_Mm1 elements were occupied by CTCF in the other three species of Muridae (2\%-5\%, Fisher's exact test, $P<$ $10^{-6}$ ). These results suggest that a B2_Mm1 element carrying an active CTCF binding site has expanded in a species-specific manner in Mus caroli.

Notably, the SINE B2_Mm1 family became active specifically in the mouse lineages after the rat-mouse divergence because fewer than 50 B2_Mm1 loci are present in the rat genome. Since the 
A

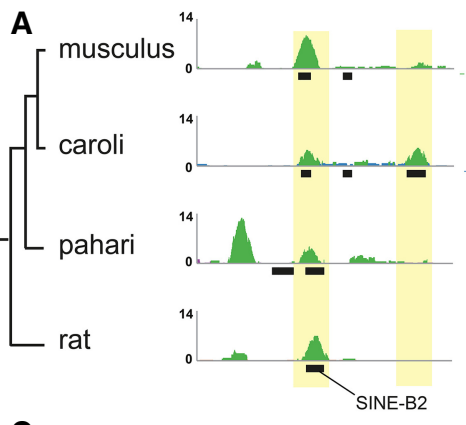

B

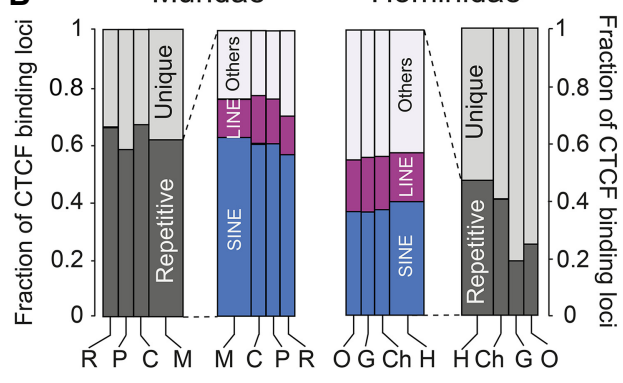

C

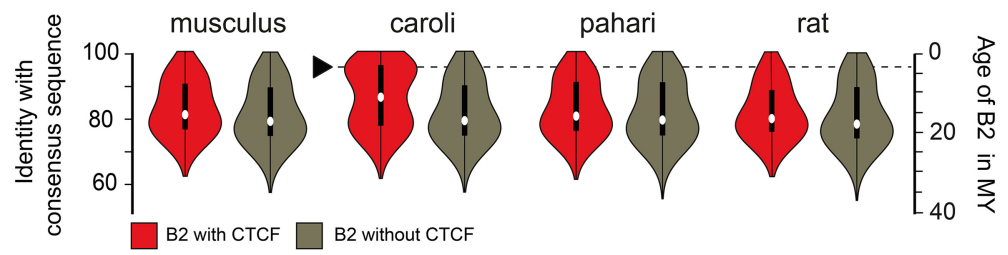

D

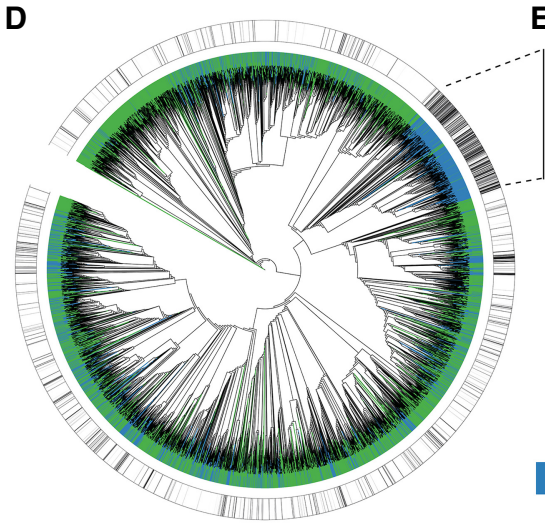

E Clade specific mutation CACTCCAGAAGAGGGCGCCA Caroli bound CACTCCAGAAGAGGGCGTCA Ancestral

Canonical CTCF binding motif

Mus caroli

Other Mus

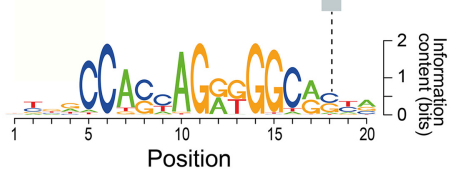
Bind CTCF

Figure 4. A single nucleotide mutation in a Mus caroli-specific expanding SINE B2 element contributed to the creation of thousands of novel CTCF binding events. (A) CTCF occupancy in the genome is shown by green tracks. The black squares show the location of SINE B2 retrotransposons. The yellow boxes represent two examples of a SINE B2 occupied by CTCF. (B) Fraction of transposable elements with CTCF binding in both Muridae (left) and Hominidae (right): (M) Mus musculus; (C) Mus caroli; (P) Mus pahari; (R) rat; (H) human; (Ch) chimpanzee; (G) gorilla; (O) orangutan. (C) Identity plots of SINE B2 with their consensus sequence, either occupied by CTCF (red) or not (brown) (Supplemental Methods SM4.1). The black arrow indicates a recent wave of SINE B2 expansion carrying CTCF binding sites in Mus caroli. (D) Neighbor-joining tree of SINE B2_Mm1 sequences from the three Mus species. The blue branches represent sequences from Mus caroli. The green branches represent sequences from Mus musculus or Mus pahari. The black lines in the outside tracks indicates the presence of a CTCF binding event. (E) A single nucleotide variation exists between the ancestral CTCF binding motif carried by the SINE B2_Mm1 element (middle) and a CTCF binding motif (top) carried by the elements recently expanded in Mus caroli. This branch-specific motif is enriched in CTCF occupancy.

rat-mouse split, B2_Mm1 elements have continued to expand along all three mouse lineages independently when compared to the ancestral rodent genome. Indeed, we also found a similar overrepresentation of species-specific B2_Mm1 elements in the Mus musculus and Mus pahari genomes, but these were not associated with a CTCF binding expansion (Supplemental Fig. S12).

To understand why CTCF binding loci were expanding only in Mus caroli, we created a B2_Mm1 sequence similarity tree within all three Mus species using neighbor joining (Supplemental Methods SM5.5). This revealed a monophyletic origin for the majority (59\%) of B2_Mm1 elements occupied by CTCF in Mus caroli (Fig. 4D). This cluster is predominantly composed of Mus caroli B2_Mm1 sequences (87\%) as well as a handful of B2_Mm1 sequences from the two other Mus species. The presence of Mus mus- culus and Mus pahari B2_Mm1 sequences suggest that either representatives of this cluster existed, albeit at low copy number, in the ancestral Mus species or that there has been random mutation of B2_Mm1 sites in the other lineages. Sequence analysis suggests that this cluster is enriched in CTCF binding occupancy because of a single nucleotide difference from the ancestral sequence: specifically, a substitution of a cytosine for a thymine at the position 18 (Fig. 4E).

The mutation arose in a portion of the motif with relatively low information context, but within a triplet that is unexpectedly critical for CTCF binding (Li et al. 2017). To confirm that this new mutation increases affinity for CTCF in our data, we compared the genome-wide representation of both the ancestral trinucleotide in this part of the motif (TCA) with the observed clade-specific trinucleotide (CCA) in regions that are both bound and not bound by CTCF. We found that, when compared to all possible trinucleotides in this part of the motif, only CCA was overrepresented in the motifs bound by CTCF, whereas both TCA and CCA were overrepresented in motifs not bound by CTCF (Supplemental Fig. S13). This result was robust to whether CTCF motifs in B2_mm1 elements were included or not (Supplemental Fig. S13B). Together this implies that the cytosine to thymine substitution in position 18 is the major reason we observe increased CTCF binding affinity in the mutated B2_mm1 element. Moreover, these new CTCF sites were mostly inserted into regions surrounding existing CTCF binding sites (Supplemental Fig. S14), suggesting that compensatory turnover is not occurring.

In summary, our analysis revealed that a single nucleotide mutation has introduced enhanced CTCF binding affinity into a SINE B2 element present in the Mus ancestor. This mutated retrotransposon massively expanded in Mus caroli adding more than 2000 species-specific CTCF binding sites of a monophyletic origin in $<3 \mathrm{MY}$.

\section{Discussion}

We generated high-quality chromosome-level assemblies of the Mus caroli and Mus pahari genomes in order to compare the dynamics of genome evolution between the Hominidae and the Muridae. Combining the genomes of Mus caroli and Mus pahari with those of Mus musculus and Rattus norvegicus yields a collection of closely related Muridae genomes that are similar in phylogenetic structure and divergence times to Hominidae (human-chimpgorilla-orangutan). This enables direct comparisons of genome

\section{Genome Research}

www.genome.org 
evolutionary dynamics between humans and their most important mammalian models.

Our results provide a detailed description of the remarkably rapid evolution of the Muridae genomes compared to Hominidae within a similar time window. Although the genome-wide increased nucleotide divergence in the Muridae lineage was previously known (Mouse Genome Sequencing Consortium 2002; Rat Genome Sequencing Project Consortium 2004), our analysis shows that all categories of genomic annotation and function have similar relative acceleration when compared to Hominidae. Indeed, our results are likely to be more precise due to the progressive increase in genome assembly quality for human and mouse over the last $10-15 \mathrm{yr}$, especially within the repetitive regions (Church et al. 2009; Schneider et al. 2017). The rate change between the two clades is similar, regardless of whether the genomic region is under evolutionary constraint (e.g., coding exons) or apparently evolving neutrally (e.g., ancestral repeats). Thus, the entire genomic system-including coding, regulatory and neutral DNA-is evolutionarily coupled, implying that differences in mutation fixation rate should largely explain the observed acceleration in Muridae.

Although the generation time of Muridae is much shorter than that of Hominidae (Li et al. 1996), this difference alone cannot fully explain the difference between evolutionary rates that we observe. Specifically, wild Muridae have a generation time of $\sim 0.5$ yr (Phifer-Rixey and Nachman 2015), but in Hominidae, it is between 20 and $30 \mathrm{yr}$ (Langergraber et al. 2012). This ratio of generation time (40-60) is much higher than the observed ratio of evolutionary rate (6-7), suggesting an important contribution from factors other than generation time (Bromham 2009) predicting either a faster rate in Hominidae or a lower rate in Muridae. We can reduce the effect of generation time by half by considering the increased rate of mutation accumulation per generation in the genome of Hominidae (Uchimura et al. 2015). A further consideration is the effective population size, which is at least one order of magnitude larger in the Muridae compared to the Hominidae (Geraldes et al. 2011; Schrago 2014). Effective population size is a critical parameter to define the mutation fixation rate in a population (Charlesworth 2009). Taken together, we can estimate the effect of population size on the increased mutation fixation rate in Hominidae compared to Muridae to an upper limit of a factor of four. However, considering the complexity of factors influencing the observed evolutionary rate, we cannot exclude other factors such as potential variation in evolutionary rates within the lineage histories that could explain part of these differences.

Our analysis also revealed a different dynamic of karyotype evolution between Muridae and Hominidae. Although the Hominidae karyotypes have remained very stable over the last 15 MY (Ferguson-Smith and Trifonov 2007), within a similar period of time, Muridae were subject to punctuate periods of accelerated karyotype instability interspersed with periods of more typical stability. These periods of karyotype instability co-occur with specific LTR repeat insertion at chromosomal breakpoints. Our analysis indicates that the rat karyotype is closer to the Murinae ancestor, which confirms previous suggestions (Zhao et al. 2004). Several studies suggest that karyotype differentiation is a direct cause of speciation (Kandul et al. 2007; Garagna et al. 2014). Moreover, a strong link has been made between explosive speciation and periods of karyotype instability in various lineages (Dobigny et al. 2017). In the Mus lineage, the Nannomys subgenus includes the highest number of species and greatest karyotype diversity (Chevret et al. 2014). Interestingly, the Nannomys di- verged from the Mus musculus lineage between the Mus caroli and Mus pahari splits (Veyrunes et al. 2005, 2006), i.e., in the same window of increased karyotype instability that we describe here.

Additionally, the analysis of transposable element activity in Muridae and Hominidae has shown that the three main classes of retrotransposons are active in both lineages. This activity has varied over time, and each lineage was subject at some point in their evolutionary history to lineage-specific bursts of retrotransposon activity. For instance, LINE elements had a recent expansive burst specifically in Muridae (Mouse Genome Sequencing Consortium 2002) that is likely still active today. Indeed, the LINE retrotransposon content, even in inbred laboratory mouse strains, can vary substantially (Nellaker et al. 2012; Lilue et al. 2018). We observed two different functional consequences of repeat-driven lineagespecific genome evolution. First, the progressive expansion of the $A b p$ gene cluster across Muridae was correlated with an enrichment of LINE and LTR elements (Janoušek et al. 2016). These retrotransposons increase local genome homology and mediate segmental duplication via nonallelic homologous recombination (Janoušek et al. 2013; Startek et al. 2015), leading to gene expansion. The $A b p$ gene cluster is involved in mating preference within the peripatric hybrid zone, where two mouse subspecies make secondary contact (Bimova et al. 2011). Together, this suggests that transposable elements are involved in the genomic mechanisms driving reproductive isolation between Mus subspecies in hybrid zones.

Another observed consequence of repeat-driven lineage-specific evolution has been the species-specific expansion of CTCF occupancy sites across the Mus caroli genome. Indeed, we demonstrated the effect of a single nucleotide substitution in a SINE B2 followed by expansion of this element to rapidly create thousands of new Mus caroli-specific CTCF binding locations. The interplay between nucleotide variation and transposition is a powerful evolutionary mechanism that can disrupt and remodel species-specific regulatory programs (Kunarso et al. 2010; Schmidt et al. 2012; Mita and Boeke 2016).

We demonstrate that comparing multiple, closely related genomes is one of the most powerful approaches to understand the biology and evolution of a single species. As the number of sequenced genomes rapidly expands in the next $10 \mathrm{yr}$ (Koepfli et al. 2015), the analysis strategy used here for the Mus caroli and Mus pahari genomes and the comparative analysis between Muridae and Homidae can be applied to diverse clades.

\section{Methods}

\section{Sequencing and assembly of Mus caroli and Mus pahari genomes}

Genomic DNA was extracted from one Mus caroli CAROLI/EiJ and one Mus pahari/EiJ female using Invitrogen's Easy-DNA kit (K180001). Following Gnerre et al. (2011), 180-bp overlapping paired-end libraries were prepared, and following Park et al. (2013), 3-kb matepair libraries were prepared. These libraries were sequenced using the Illumina HiSeq 2000 platform. The reads were assembled into contigs and scaffolds using the ALLPATHS-LG assembler (Gnerre et al. 2011). High molecular weight DNA was extracted from Mus caroli/EiJ and Mus pahari/EiJ following the protocol in Supplemental Methods SM1.2 to construct an optical map using the OpGen platform. The OpGen Genome-Builder software was used to assemble the NGS scaffolds into super scaffolds based on the optical map. Super scaffolds and scaffolds were assembled into pseudochromosomes with Ragout (Kolmogorov et al. 2016). 
To guide the assembly, Ragout used a multiple alignment constructed with Progressive Cactus (Paten et al. 2011). This alignment included the scaffolds of Mus caroli, Mus Pahari, and the genomes of Mus musculus (C57BL/6NJ GRCm38/mm10 assembly) and Rattus norvegicus V5.0. See Supplemental Methods SM1.1SM1.5 for more details.

\section{Gene annotation}

Mus caroli and Mus pahari genes were annotated using a combination of three annotation pipelines: TransMap (Stanke et al. 2008), AUGUSTUS (Stanke et al. 2006), and a new mode of AUGUSTUS called Comparative AUGUSTUS (AUGUSTUS-CGP) (Konig et al. 2016). The GENCODE set of Mus musculus transcripts (M8 release) (Harrow et al. 2012) was used with the TransMap pipeline. In addition, RNA-seq data was used with the AUGUSTUS and AUGUSTUSCGP pipelines. To prepare the RNA-seq data, RNA was extracted from multiple tissues (brain, liver, heart, kidney) from Mus caroli and Mus pahari using Qiagen's RNeasy kit following the manufacturer's instructions. RNA-seq libraries were generated with Illumina's TruSeq Ribo-Zero strand-specific kit and then sequenced on the Illumina HiSeq 2000 platform with 100-bp paired-end reads. The annotation of the $A b p$ gene clusters was refined with a combination of BLAST (Altschul et al. 1990), hmmsearch (Finn et al. 2011), and exonerate (Slater and Birney 2005). The relationship between the $S c g b$ and $A b p$ nomenclatures is described earlier. See Supplemental Methods SM1.7 and SM4.5 for more details.

\section{Divergence time estimation}

The divergence times of Mus musculus from Mus caroli and Mus pahari was estimated based on a set of fourfold degenerate sites from amino acids conserved across all mammals. Three different subsets of fourfold degenerate sites with similar size were created based on (1) random selection; (2) tissues-specific genes; and (3) housekeeping genes. BEAST 2 (Bouckaert et al. 2014) was used to infer the divergence time independently with the three data sets of fourfold degenerate sites and different evolutionary models (calibrated Yule model, Birth-Death Model, GTR, HKY85, strict clock, uncorrelated relaxed clock). Fossil record information of the mouse-rat divergence (Jacobs and Flynn 2005) was used to calibrate the molecular clock in all our analyses. See Supplemental Methods SM1.16 for more details.

\section{Chromosome rearrangement analysis}

The synteny breaks involving large genomic regions among Mus musculus, Mus caroli, and Mus pahari were identified with the reciprocal cross-species chromosome painting experiments described in Supplemental Methods SM1.3. To further define the evolutionarily syntenic breakpoints on the chromosomes of the C57BL/6J strain between Mus musculus and Mus pahari, a Mouse CGH (244k) microarray was used with the chromosome-specific DNA libraries of Mus pahari. The Mouse CGH array was analyzed using the CGHweb tool (Lai et al. 2008), with default parameters. For the comparison between Mus musculus and rat and between all four Hominidaes, inter-chromosomal synteny breaks involving genomic regions longer than $3 \mathrm{Mb}$ were identified and selected using the synteny map in Ensembl v82 (Aken et al. 2017).

To estimate the rate of inter-chromosomal rearrangements in each clade, we created a distance matrix based on the number of synteny breaks. The matrix was used to compute a neighbor-joining tree. The branch length from the resulting tree represents an estimation of the number of synteny breaks that occurred in the branch (Fig. 1C).
Repeat enrichment in a \pm 40 -Mb region around the breakpoints was analyzed by counting the occurrence of each repeat element in $200-\mathrm{kb}$ sliding windows and averaging over all breakpoints. For each averaged window, a $Z$-score was calculated based on the $80-\mathrm{Mb}$ region analyzed (excluding the $\pm 2-\mathrm{Mb}$ region around the breakpoint). The size of $\pm 40 \mathrm{Mb}$ was chosen because it is the longest possible region that does not include a start or end of a chromosome. We evaluated statistical significance of the repeat enrichment by calculating an empirical $P$-value by 1,000,000 comparisons of the observed number of repeat elements in a \pm 2 -Mb region centered on the breakpoint with an equivalent number of random regions.

See Supplemental Methods SM2 for more details.

\section{Evolutionary rate analysis}

The nucleotide sequence divergence between Mus musculus and the other three murid species, as well as between human and other Hominidae, was estimated from LASTZ pairwise alignments following the Ensembl methodology (Herrero et al. 2016). For each clade and each genomic class, the value of the nucleotide divergence against the divergence time was plotted for each pair of species involved in the comparison. The rate of nucleotide divergence from each clade was derived from a linear regression. An ANCOVA test was used to evaluate the statistical significance of the difference of rates between each genomic category, with the rate as response variable and the genomic category as a fixed factor.

The rate of unshared genomic segments between Mus musculus and other Muridae as well as between human and other Hominidae was estimated from LASTZ pairwise alignments as defined above. A genomic region was defined as shared between two species if the region had an alignment between the two species with $<50 \%$ of gapped sequence. For each clade and each genomic class, the value of the unshared genomic segments was plotted against the divergence time for each pair of species involved in the comparison. The turnover of genomic segments from each clade was derived from a linear regression. An ANCOVA test was used for evaluation of the statistical significance of the difference of turnover between each genomic category, again with turnover rate as response and the genomic category as a fixed factor.

See Supplemental Methods SM3 for more details.

\section{Repeat analysis}

Repeat elements were identified with RepeatMasker 3.2.8 (Smit et al. 1996-2010) using the rodent repeat libraries for the four Muridae genomes and the primate repeat library for the four Hominidae genomes. Simple repeats and microsatellite elements were removed. Fragmented hits identified by RepeatMasker as belonging to a same repeat were merged. The age of each repeat element was estimated as

$$
t=d / r_{\text {class }}
$$

where $d$ is the sequence identity of the repeat with its consensus sequence, and $r_{\text {class }}$ is the nucleotide evolutionary rate of the repeat class. The rate was calculated from the ancestral repeats (i.e., repeated elements shared between the four Muridae or the four Hominidae genomes). See Supplemental Methods SM4 for more details.

\section{Retrocopy analysis}

Retrocopies in the Muridae and Hominidae genomes were detected as previously described (Navarro and Galante 2013). In order to comprehensively annotate retrocopies in Mus musculus and Homo sapiens, we used a combination of manual and automatic curation

\section{Genome Research}

www.genome.org 
workflows. We considered the manually annotated processed pseudogenes from GENCODE M13 and v24, respectively (Pei et al. 2012), and processed pseudogenes from pseudopipe (Zhang et al. 2006; Sisu et al. 2014). Mature transcript sequences were derived from Ensembl v86 and aligned to the corresponding reference genome using BLAT (mask=lower; -tileSize=12; -minIdentity=75; -minScore=100). The age of each retrocopy was estimated as

$$
t=2 d /\left(r_{\text {parent }}+r_{\text {retrocopy }}\right)
$$

where $d$ is the sequence identity between a retrocopy and its parental gene; $r_{\text {parent }}$ is the nucleotide evolutionary rate of the parental gene defined from the set of one-to-one gene orthologs shared between the four Muridae or four Hominidae; and $r_{\text {retrocopy }}$ is the nucleotide evolutionary rate of the retrocopies calculated from the retrocopies shared between the four Muridae or the four Hominidae. See Supplemental Methods SM4 for more details.

\section{CTCF binding site analysis}

We profiled the binding of CTCF in livers of Mus musculus C57BL/ 6J, Mus caroli CAROLI/EiJ, Mus pahari/EiJ, and Rattus norvegicus using the ChIP-seq protocol described in Schmidt et al. (2009). The paired-end libraries were sequenced at $100 \mathrm{bp}$ on the HiSeq2000 platform. In addition, the data set from Schwalie et al (2013) was used to identify the CTCF binding sites in primates. Sequencing reads were aligned to the appropriate reference genome using Bowtie 2 version 2.2.6 (Langmead and Salzberg 2012). MACS version 1.4.2 (Zhang et al. 2008) was used with a $P$-value threshold of 0.001 to call read enrichment representing CTCF binding sites. Peaks present in at least two biological replicates were used for the analysis. The binding motif in each CTCF binding region was identified with the FIMO program from the MEME suite version 4.10.2 (Bailey et al. 2015) and using the CTCF position weight matrix (CTCF.p2) from the SwissRegulon database (Pachkov et al. 2013). See Supplemental Methods SM1.11 and SM5 for more details.

\section{SINE B2_Mm1 neighbor-joining classification}

SINE B2_Mm1 sequences from the three Mus species were selected after filtering out sequences with the following characteristics (1) shorter than $150 \mathrm{bp}$; (2) at least one unknown nucleotide (N); and (3) $>10 \%$ of substitution, insertion, or deletion with the SINE B2_Mm1 consensus sequence. The sequences were aligned using MAFFT version 7.222 (Katoh and Standley 2013), and the alignment was used to calculate a neighbor-joining tree using FastTree version 2.1.9 (Price et al. 2010) with local bootstrap and minimum-evolution model. The ancestral sequence of the B2_Mm1 CTCF binding motif was inferred using FASTML (Ashkenazy et al. 2012), with the neighbor-joining method and the JC model. A second independent approach based on PRANK (Loytynoja and Goldman 2010), with the options -showanc -keep -njtree, was used to confirm the ancestral sequence inference. See Supplemental Methods SM5.5-SM5.7 for more details.

\section{Data access}

The genome assemblies of Mus caroli and Mus pahari from this study have been submitted to the European Nucleotide Archive (ENA; https://www.ebi.ac.uk/ena) under accession numbers GCA_900094665 (Mus caroli) and GCA_900095145 (Mus pahari). All reads from the ChIP-seq and RNA-seq experiments in this study have been submitted to ArrayExpress (https://www.ebi.ac.uk/ arrayexpress) under accession numbers E-MTAB-5768 (RNA-seq) and E-MTAB-5769 (ChIP-seq). A supplemental web page with links to raw data and other information is available at http://www.ebi.ac .uk/research/flicek/publications/FOG21.

\section{Acknowledgments}

This project was supported by the Wellcome Trust (grant numbers WT108749/Z/15/Z, WT098051, WT202878/Z/16/Z, and WT202878/B/16/Z), the National Human Genome Research Institute (U41HG007234), Cancer Research UK (20412), the European Research Council (615584), the Biotechnology and Biological Sciences Research Council (BB/N02317X/a), and the European Molecular Biology Laboratory. The research leading to these results has received funding from the European Community's Seventh Framework Programme (FP7/2010-2014) under grant agreement 244356 (NextGen) and from the European Union's Seventh Framework Programme (FP7/2007-2013) under grant agreement HEALTH-F4-2010-241504 (EURATRANS). We thank the genomics, bioinformatics, and BRU cores at the CRUK Cambridge Institute for technical support, the sequencing facilities at the Wellcome Sanger Institute, and computational support from EMBL-EBI and WSI as well as the Conservatoire Génétique de la Souris Sauvage (ISEM, France) and Plateforme Cytogénomique évolutive of the LabEx CeMEB. We also thank Bee Ling N, Beiyuan Fu, Sandra Louzada, and Mark Simmonds for assistance in chromosome sorting, chromosome painting, and array painting.

Author contributions: Study design, project leadership, and manuscript writing were done by D.T., D.T.O., and P.F.; genome sequencing and assembly were the responsibility of I.S., M.K., D.T., A.D., S.A., K.S., A.Z., M.D., M.A.Q., W.C., L.J., L.G., S.P., K.H., M.G., L.C., and T.M.K.; comparative genomics and genome annotation were performed by I.F., M.S., S.N., B.P., C.C., M.M., W.A., B.A., and F.M.; D.M.G., D.T., A.A.J., and V.C. completed the evolutionary analysis; C.V.O. and B.W. were responsible for the introgression analysis; chromosome rearrangements analysis was done by D.T. and F.Y.; D.T. did repeat analysis; F.C.P.N., D.T., C.S., and M.G. did the retrocopy analysis; CTCF and repeat analysis were done by M.R., C.F., D.T., and M.H.; Abp region analysis was done by V.J., G.Y., R.C.K., and C.M.L.; and F.V., D.J.A., and A.B. were responsible for reagent supply.

\section{References}

Aken BL, Achuthan P, Akanni W, Amode MR, Bernsdorff F, Bhai J, Billis K, Carvalho-Silva D, Cummins C, Clapham P, et al. 2017. Ensembl 2017. Nucleic Acids Res 45: D635-D642.

Altschul SF, Gish W, Miller W, Myers EW, Lipman DJ. 1990. Basic local alignment search tool. J Mol Biol 215: 403-410.

Ashkenazy H, Penn O, Doron-Faigenboim A, Cohen O, Cannarozzi G, Zomer O, Pupko T. 2012. FastML: a web server for probabilistic reconstruction of ancestral sequences. Nucleic Acids Res 40: W580-W584.

Atanur SS, Diaz AG, Maratou K, Sarkis A, Rotival M, Game L, Tschannen MR, Kaisaki PJ, Otto GW, Ma MC, et al. 2013. Genome sequencing reveals loci under artificial selection that underlie disease phenotypes in the laboratory rat. Cell 154: 691-703.

Bailey JA, Baertsch R, Kent WJ, Haussler D, Eichler EE. 2004. Hotspots of mammalian chromosomal evolution. Genome Biol 5: R23.

Bailey TL, Johnson J, Grant CE, Noble WS. 2015. The MEME suite. Nucleic Acids Res 43: W39-W49.

Batzer MA, Deininger PL. 2002. Alu repeats and human genomic diversity. Nat Rev Genet 3: 370-379.

Bimova BV, Macholan M, Baird SJ, Munclinger P, Dufkova P, Laukaitis CM, Karn RC, Luzynski K, Tucker PK, Pialek J. 2011. Reinforcement selection acting on the European house mouse hybrid zone. Mol Ecol 20: 2403-2424.

Bouckaert R, Heled J, Kuhnert D, Vaughan T, Wu CH, Xie D, Suchard MA Rambaut A, Drummond AJ. 2014. BEAST 2: a software platform for Bayesian evolutionary analysis. PLoS Comput Biol 10: e1003537.

Bourque G, Leong B, Vega VB, Chen X, Lee YL, Srinivasan KG, Chew JL, Ruan Y, Wei CL, Ng HH, et al. 2008. Evolution of the mammalian 
transcription factor binding repertoire via transposable elements. Genome Res 18: 1752-1762.

The Bovine Genome Sequencing and Analysis Consortium. 2009. The genome sequence of taurine cattle: a window to ruminant biology and evolution. Science 324: 522-528.

Bromham L. 2009. Why do species vary in their rate of molecular evolution? Biol Lett 5: 401-404.

Capilla L, Sánchez-Guillén RA, Farré M, Paytuví-Gallart A, Malinverni R, Ventura J, Larkin DM, Ruiz-Herrera A. 2016. Mammalian comparative genomics reveals genetic and epigenetic features associated with genome reshuffling in Rodentia. Genome Biol Evol 8: 3703-3717.

Carbone L, Harris RA, Gnerre S, Veeramah KR, Lorente-Galdos B, Huddleston J, Meyer TJ, Herrero J, Roos C, Aken B, et al. 2014. Gibbon genome and the fast karyotype evolution of small apes. Nature 513: 195-201.

Charlesworth B. 2009. Fundamental concepts in genetics: effective population size and patterns of molecular evolution and variation. Nat Rev Genet 10: 195-205.

Chevret P, Robinson TJ, Perez J, Veyrunes F, Britton-Davidian J. 2014. A phylogeographic survey of the pygmy mouse Mus minutoides in South Africa: taxonomic and karyotypic inference from cytochrome $b$ sequences of museum specimens. PLoS One 9: e98499.

The Chimpanzee Sequencing and Analysis Consortium. 2005. Initial sequence of the chimpanzee genome and comparison with the human genome. Nature 437: 69-87.

Chuong EB, Elde NC, Feschotte C. 2016. Regulatory evolution of innate immunity through co-option of endogenous retroviruses. Science $\mathbf{3 5 1}$ : 1083-1087.

Church DM, Goodstadt L, Hillier LW, Zody MC, Goldstein S, She X, Bult CJ, Agarwala R, Cherry JL, DiCuccio M, et al. 2009. Lineage-specific biology revealed by a finished genome assembly of the mouse. PLoS Biol 7: e1000112.

Dobigny G, Britton-Davidian J, Robinson TJ. 2017. Chromosomal polymorphism in mammals: an evolutionary perspective. Biol Rev Camb Philos Soc 92: 1-21.

Esnault C, Maestre J, Heidmann T. 2000. Human LINE retrotransposons generate processed pseudogenes. Nat Genet 24: 363-367.

Ferguson-Smith MA, Trifonov V. 2007. Mammalian karyotype evolution. Nat Rev Genet 8: 950-962.

Finn RD, Clements J, Eddy SR. 2011. HMMER web server: interactive sequence similarity searching. Nucleic Acids Res 39: W29-W37.

Foote AD, Liu Y, Thomas GW, Vinar T, Alfoldi J, Deng J, Dugan S, van Elk CE, Hunter ME, Joshi V, et al. 2015. Convergent evolution of the genomes of marine mammals. Nat Genet 47: 272-275.

Gaffney DJ, Keightley PD. 2006. Genomic selective constraints in murid noncoding DNA. PLoS Genet 2: e204

Garagna S, Page J, Fernandez-Donoso R, Zuccotti M, Searle JB. 2014. The Robertsonian phenomenon in the house mouse: mutation, meiosis and speciation. Chromosoma 123: 529-544.

Gazave E, Darre F, Morcillo-Suarez C, Petit-Marty N, Carreno A, Marigorta UM, Ryder OA, Blancher A, Rocchi M, Bosch E, et al. 2011. Copy number variation analysis in the great apes reveals species-specific patterns of structural variation. Genome Res 21: 1626-1639.

Geraldes A, Basset P, Smith KL, Nachman MW. 2011. Higher differentiation among subspecies of the house mouse (Mus musculus) in genomic regions with low recombination. Mol Ecol 20: 4722-4736.

Gnerre S, Maccallum I, Przybylski D, Ribeiro FJ, Burton JN, Walker BJ, Sharpe T, Hall G, Shea TP, Sykes S, et al. 2011. High-quality draft assemblies of mammalian genomes from massively parallel sequence data. Proc Natl Acad Sci 108: 1513-1518.

Goetting-Minesky MP, Makova KD. 2006. Mammalian male mutation bias: impacts of generation time and regional variation in substitution rates. Mol Evol 63: 537-544.

Gordon D, Huddleston J, Chaisson MJ, Hill CM, Kronenberg ZN, Munson KM, Malig M, Raja A, Fiddes I, Hillier LW, et al. 2016. Long-read sequence assembly of the gorilla genome. Science 352: aae0344.

Groenen MA, Archibald AL, Uenishi H, Tuggle CK, Takeuchi Y, Rothschild MF, Rogel-Gaillard C, Park C, Milan D, Megens HJ, et al. 2012. Analyses of pig genomes provide insight into porcine demography and evolution. Nature 491: 393-398.

Harrow J, Frankish A, Gonzalez JM, Tapanari E, Diekhans M, Kokocinski F, Aken BL, Barrell D, Zadissa A, Searle S, et al. 2012. GENCODE: the reference human genome annotation for The ENCODE Project. Genome Res 22: $1760-1774$.

Hedges DJ, Deininger PL. 2007. Inviting instability: transposable elements, double-strand breaks, and the maintenance of genome integrity. Mutat Res 616: 46-59.

Herrero J, Muffato M, Beal K, Fitzgerald S, Gordon L, Pignatelli M, Vilella AJ, Searle SM, Amode R, Brent S, et al. 2016. Ensembl comparative genomics resources. Database (Oxford) 2016: bav096.
Irie M, Koga A, Kaneko-Ishino T, Ishino F. 2016. An LTR retrotransposon-derived gene displays lineage-specific structural and putative species-specific functional variations in eutherians. Front Chem 4: 26.

Jacobs L, Flynn L. 2005. Of mice... again: the Siwalik rodent record, murine distribution, and molecular clocks. In Interpreting the past: essays on human, primate, and mammal evolution in honor of David Pilbeam (ed. Lieberman D, et al.), pp. 63-80. Brill Academic Publishers, Boston.

Jacques PE, Jeyakani J, Bourque G. 2013. The majority of primate-specific regulatory sequences are derived from transposable elements. PLoS Genet 9: e1003504.

Janoušek V, Karn RC, Laukaitis CM. 2013. The role of retrotransposons in gene family expansions: insights from the mouse $A b p$ gene family. BMC Evol Biol 13: 107.

Janoušek V, Laukaitis CM, Yanchukov A, Karn RC. 2016. The role of retrotransposons in gene family expansions in the human and mouse genomes. Genome Biol Evol 8: 2632-2650.

Johnson DS, Mortazavi A, Myers RM, Wold B. 2007. Genome-wide mapping of in vivo protein-DNA interactions. Science 316: 1497-1502.

Kandul NP, Lukhtanov VA, Pierce NE. 2007. Karyotypic diversity and speciation in Agrodiaetus butterflies. Evolution 61: 546-559.

Kass DH, Kim J, Rao A, Deininger PL. 1997. Evolution of B2 repeats: the muroid explosion. Genetica 99: 1-13.

Katoh K, Standley DM. 2013. MAFFT multiple sequence alignment software version 7: improvements in performance and usability. Mol Biol Evol 30: 772-780.

Kim EB, Fang X, Fushan AA, Huang Z, Lobanov AV, Han L, Marino SM, Sun $X$, Turanov AA, Yang P, et al. 2011. Genome sequencing reveals insights into physiology and longevity of the naked mole rat. Nature 479: 223-227.

Kirkness EF, Bafna V, Halpern AL, Levy S, Remington K, Rusch DB, Delcher $\mathrm{AL}$, Pop M, Wang W, Fraser CM, et al. 2003. The dog genome: survey sequencing and comparative analysis. Science 301: 1898-1903.

Koepfli KP, Paten B, Genome 10K Community of Scientists, O'Brien SJ. 2015. The Genome 10K Project: a way forward. Annu Rev Anim Biosci 3: $57-111$.

Kolmogorov M, Armstrong J, Raney BJ, Streeter I, Dunn M, Yang F, Odom D, Flicek P, Keane T, Thybert D, et al. 2016. Chromosome assembly of large and complex genomes using multiple references. bioRxiv doi:10.1101/ 088435.

Konig S, Romoth LW, Gerischer L, Stanke M. 2016. Simultaneous gene finding in multiple genomes. Bioinformatics 32: 3388-3395.

Kunarso G, Chia NY, Jeyakani J, Hwang C, Lu X, Chan YS, Ng HH, Bourque G. 2010. Transposable elements have rewired the core regulatory network of human embryonic stem cells. Nat Genet 42: 631-634.

Lai W, Choudhary V, Park PJ. 2008. CGHweb: a tool for comparing DNA copy number segmentations from multiple algorithms. Bioinformatics 24: $1014-1015$.

Langergraber KE, Prufer K, Rowney C, Boesch C, Crockford C, Fawcett K, Inoue $\mathrm{E}$, Inoue-Muruyama M, Mitani JC, Muller MN, et al. 2012. Generation times in wild chimpanzees and gorillas suggest earlier divergence times in great ape and human evolution. Proc Natl Acad Sci 109: 15716-15721.

Langmead B, Salzberg SL. 2012. Fast gapped-read alignment with Bowtie 2. Nat Methods 9: 357-359.

Laukaitis C, Karn RC. 2012. Recognition of subspecies status mediated by androgen-binding protein (ABP) in the evolution of incipient reinforcement on the European house mouse hybrid zone. In Evolution of the house mouse (ed. Macholan M, et al.), pp. 150-190. Cambridge University Press, Cambridge, UK.

Laukaitis CM, Heger A, Blakley TD, Munclinger P, Ponting CP, Karn RC. 2008. Rapid bursts of androgen-binding protein ( $A b p$ ) gene duplication occurred independently in diverse mammals. BMC Evol Biol 8: 46.

Li WH, Tanimura M. 1987. The molecular clock runs more slowly in man than in apes and monkeys. Nature 326: 93-96.

Li WH, Ellsworth DL, Krushkal J, Chang BH, Hewett-Emmett D. 1996. Rates of nucleotide substitution in primates and rodents and the generationtime effect hypothesis. Mol Phylogenet Evol 5: 182-187.

Li W, Shang L, Huang K, Li J, Wang Z, Yao H. 2017. Identification of critical base pairs required for CTCF binding in motif M1 and M2. Protein Cell 8: 544-549.

Lilue J, Doran AG, Fiddes IT, Abrudan M, Armstrong J, Bennet R, Chow W, Collins J, Czechanski A, Danecek P, et al. 2018. Multiple laboratory mouse reference genomes define strain specific haplotypes and novel functional loci. bioRxiv doi: 10.1101/235838.

Lin L, Shen S, Tye A, Cai JJ, Jiang P, Davidson BL, Xing Y. 2008. Diverse splicing patterns of exonized Alu elements in human tissues. PLoS Genet 4: e1000225

Lindblad-Toh K, Wade CM, Mikkelsen TS, Karlsson EK, Jaffe DB, Kamal M, Clamp M, Chang JL, Kulbokas EJ III, Zody MC, et al. 2005. Genome sequence, comparative analysis and haplotype structure of the domestic dog. Nature 438: 803-819.

\section{Genome Research}

www.genome.org 
Lindblad-Toh K, Garber M, Zuk O, Lin MF, Parker BJ, Washietl S, Kheradpour P, Ernst J, Jordan G, Mauceli E, et al. 2011. A high-resolution map of human evolutionary constraint using 29 mammals. Nature 478: 476-482.

Liu GE, Alkan C, Jiang L, Zhao S, Eichler EE. 2009. Comparative analysis of Alu repeats in primate genomes. Genome Res 19: 876-885.

Liu S, Lorenzen ED, Fumagalli M, Li B, Harris K, Xiong Z, Zhou L, Korneliussen TS, Somel M, Babbitt C, et al. 2014. Population genomics reveal recent speciation and rapid evolutionary adaptation in polar bears. Cell 157: 785-794.

Locke DP, Hillier LW, Warren WC, Worley KC, Nazareth LV, Muzny DM, Yang SP, Wang Z, Chinwalla AT, Minx P, et al. 2011. Comparative and demographic analysis of orang-utan genomes. Nature 469: 529-533.

Loytynoja A, Goldman N. 2010. webPRANK: a phylogeny-aware multiple sequence aligner with interactive alignment browser. BMC Bioinformatics 11: 579 .

Lynch VJ, Leclerc RD, May G, Wagner GP. 2011. Transposon-mediated rewiring of gene regulatory networks contributed to the evolution of pregnancy in mammals. Nat Genet 43: 1154-1159.

Marques-Bonet T, Kidd JM, Ventura M, Graves TA, Cheng Z, Hillier LW, Jiang Z, Baker C, Malfavon-Borja R, Fulton LA, et al. 2009. A burst of segmental duplications in the genome of the African great ape ancestor. Nature 457: 877-881.

Mita P, Boeke JD. 2016. How retrotransposons shape genome regulation. Curr Opin Genet Dev 37: 90-100.

Mortazavi A, Leeper Thompson EC, Garcia ST, Myers RM, Wold B. 2006 Comparative genomics modeling of the NRSF/REST repressor network: from single conserved sites to genome-wide repertoire. Genome Res 16: 1208-1221.

Mouse Genome Sequencing Consortium. 2002. Initial sequencing and comparative analysis of the mouse genome. Nature 420: 520-562.

Murphy WJ, Larkin DM, Everts-van der Wind A, Bourque G, Tesler G, Auvil L, Beever JE, Chowdhary BP, Galibert F, Gatzke L, et al. 2005. Dynamics of mammalian chromosome evolution inferred from multispecies comparative maps. Science 309: 613-617.

Navarro FC, Galante PA. 2013. RCPedia: a database of retrocopied genes. Bioinformatics 29: 1235-1237.

Navarro FC, Galante PA. 2015. A genome-wide landscape of retrocopies in primate genomes. Genome Biol Evol 7: 2265-2275.

Nellaker C, Keane TM, Yalcin B, Wong K, Agam A, Belgard TG, Flint J, Adams DJ, Frankel WN, Ponting CP. 2012. The genomic landscape shaped by selection on transposable elements across 18 mouse strains. Genome Biol 13: R45.

Pace JK II, Feschotte C. 2007. The evolutionary history of human DNA transposons: evidence for intense activity in the primate lineage. Genome Res 17: $422-432$.

Pachkov M, Balwierz PJ, Arnold P, Ozonov E, van Nimwegen E. 2013. SwissRegulon, a database of genome-wide annotations of regulatory sites: recent updates. Nucleic Acids Res 41: D214-D220.

Park N, Shirley L, Gu Y, Keane T, Swerdlow H, Quail M. 2013. An improved approach to mate-paired library preparation for Illumina sequencing. Methods Next Generation Seq 1: 10-20.

Paten B, Earl D, Nguyen N, Diekhans M, Zerbino D, Haussler D. 2011. Cactus: algorithms for genome multiple sequence alignment. Genome Res 21: 1512-1528.

Pei B, Sisu C, Frankish A, Howald C, Habegger L, Mu XJ, Harte R, Balasubramanian S, Tanzer A, Diekhans M, et al. 2012. The GENCODE pseudogene resource. Genome Biol 13: R51.

Phifer-Rixey M, Nachman MW. 2015. Insights into mammalian biology from the wild house mouse Mus musculus. eLife 4. doi: 10.7554/ eLife.05959.001.

Pollard KS, Salama SR, Lambert N, Lambot MA, Coppens S, Pedersen JS, Katzman S, King B, Onodera C, Siepel A, et al. 2006. An RNA gene expressed during cortical development evolved rapidly in humans. Nature 443: 167-172.

Price MN, Dehal PS, Arkin AP. 2010. FastTree 2 - approximately maximumlikelihood trees for large alignments. PLoS One 5: e9490.

Rat Genome Sequencing Project Consortium. 2004. Genome sequence of the Brown Norway rat yields insights into mammalian evolution. Nature 428: 493-521.

Ray DA, Pagan HJ, Thompson ML, Stevens RD. 2007. Bats with hATs: evidence for recent DNA transposon activity in genus Myotis. Mol Biol Evol 24: 632-639.

Rhesus Macaque Genome Sequencing and Analysis Consortium. 2007. Evolutionary and biomedical insights from the rhesus macaque genome. Science 316: 222-234.
Robberecht C, Voet T, Zamani Esteki M, Nowakowska BA, Vermeesch JR. 2013. Nonallelic homologous recombination between retrotransposable elements is a driver of de novo unbalanced translocations. Genome Res 23: 411-418.

Scally A, Dutheil JY, Hillier LW, Jordan GE, Goodhead I, Herrero J, Hobolth A, Lappalainen T, Mailund T, Marques-Bonet T, et al. 2012. Insights into hominid evolution from the gorilla genome sequence. Nature $\mathbf{4 8 3}$ 169-175.

Schmidt D, Wilson MD, Spyrou C, Brown GD, Hadfield J, Odom DT. 2009. ChIP-seq: using high-throughput sequencing to discover protein-DNA interactions. Methods 48: 240-248.

Schmidt D, Schwalie PC, Wilson MD, Ballester B, Gonçalves A, Kutter C, Brown GD, Marshall A, Flicek P, Odom DT. 2012. Waves of retrotransposon expansion remodel genome organization and CTCF binding in multiple mammalian lineages. Cell 148: $335-348$.

Schneider VA, Graves-Lindsay T, Howe K, Bouk N, Chen HC, Kitts PA, Murphy TD, Pruitt KD, Thibaud-Nissen F, Albracht D, et al. 2017. Evaluation of GRCh38 and de novo haploid genome assemblies demonstrates the enduring quality of the reference assembly. Genome Res $\mathbf{2 7}$ 849-864.

Schrago CG. 2014. The effective population sizes of the anthropoid ancestors of the human-chimpanzee lineage provide insights on the historical biogeography of the great apes. Mol Biol Evol 31: 37-47.

Schwalie PC, Ward MC, Cain CE, Faure AJ, Gilad Y, Odom DT, Flicek P. 2013. Co-binding by YY1 identifies the transcriptionally active, highly conserved set of CTCF-bound regions in primate genomes. Genome Biol 14: R148.

Sisu C, Pei B, Leng J, Frankish A, Zhang Y, Balasubramanian S, Harte R, Wang D, Rutenberg-Schoenberg M, Clark W, et al. 2014. Comparative analysis of pseudogenes across three phyla. Proc Natl Acad Sci 111: 13361-13366.

Slater GS, Birney E. 2005. Automated generation of heuristics for biological sequence comparison. BMC Bioinformatics 6: 31 .

Smit AFA, Hubley R, Green P. 1996-2010. RepeatMasker Open-3.0. http ://www.repeatmasker.org/.

Stanke M, Tzvetkova A, Morgenstern B. 2006. AUGUSTUS at EGASP: using EST, protein and genomic alignments for improved gene prediction in the human genome. Genome Biol 7(Suppl 1): S11.1-S11.8.

Stanke M, Diekhans M, Baertsch R, Haussler D. 2008. Using native and syntenically mapped cDNA alignments to improve de novo gene finding. Bioinformatics 24: 637-644.

Startek M, Szafranski P, Gambin T, Campbell IM, Hixson P, Shaw CA Stankiewicz P, Gambin A. 2015. Genome-wide analyses of LINE-LINEmediated nonallelic homologous recombination. Nucleic Acids Res 43: 2188-2198.

Sultana T, Zamborlini A, Cristofari G, Lesage P. 2017. Integration site selection by retroviruses and transposable elements in eukaryotes. Nat Rev Genet 18: 292-308.

Uchimura A, Higuchi M, Minakuchi Y, Ohno M, Toyoda A, Fujiyama A, Miura I, Wakana S, Nishino J, Yagi T. 2015. Germline mutation rates and the long-term phenotypic effects of mutation accumulation in wild-type laboratory mice and mutator mice. Genome Res 25: 1125-1134.

Veyrunes F, Britton-Davidian J, Robinson TJ, Calvet E, Denys C, Chevret P. 2005. Molecular phylogeny of the African pygmy mice, subgenus Nannomys (Rodentia, Murinae, Mus): implications for chromosomal evolution. Mol Phylogenet Evol 36: 358-369.

Veyrunes F, Dobigny G, Yang F, O'Brien PC, Catalan J, Robinson TJ, BrittonDavidian J. 2006. Phylogenomics of the genus Mus (Rodentia; Muridae): Extensive genome repatterning is not restricted to the house mouse. Proc Biol Sci 273: 2925-2934.

Wu H, Guang X, Al-Fageeh MB, Cao J, Pan S, Zhou H, Zhang L, Abutarboush MH, Xing Y, Xie Z, et al. 2014. Camelid genomes reveal evolution and adaptation to desert environments. Nat Commun 5: 5188 .

Zhang Z, Carriero N, Zheng D, Karro J, Harrison PM, Gerstein M. 2006. PseudoPipe: an automated pseudogene identification pipeline. Bioinformatics 22: 1437-1439.

Zhang Y, Liu T, Meyer CA, Eeckhoute J, Johnson DS, Bernstein BE, Nusbaum C, Myers RM, Brown M, Li W, et al. 2008. Model-based analysis of ChIPSeq (MACS). Genome Biol 9: R137.

Zhao S, Shetty J, Hou L, Delcher A, Zhu B, Osoegawa K, de Jong P, Nierman WC, Strausberg RL, Fraser CM. 2004. Human, mouse, and rat genome large-scale rearrangements: stability versus speciation. Genome Res 14: 1851-1860.

Received December 29, 2017; accepted in revised form March 5, 2018. 


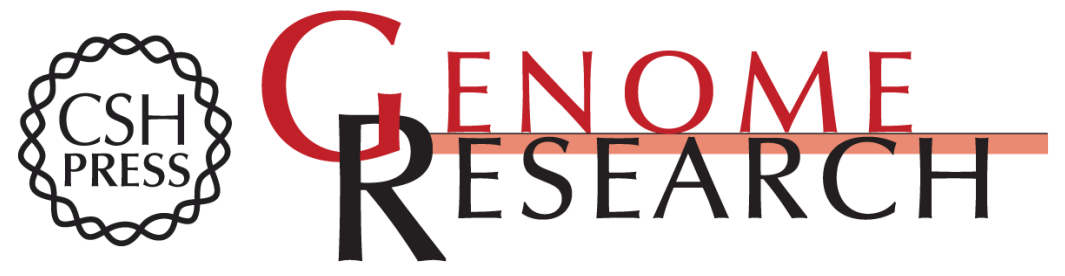

\section{Repeat associated mechanisms of genome evolution and function revealed by the Mus caroli and Mus pahari genomes}

David Thybert, Masa Roller, Fábio C.P. Navarro, et al.

Genome Res. 2018 28: 448-459 originally published online March 21, 2018

Access the most recent version at doi:10.1101/gr.234096.117

\section{Supplemental} Material

\section{References}

Open Access

Creative Commons

License

Email Alerting Service
http://genome.cshlp.org/content/suppl/2018/03/21/gr.234096.117.DC1

This article cites 104 articles, 23 of which can be accessed free at: http://genome.cshlp.org/content/28/4/448.full.html\#ref-list-1

Freely available online through the Genome Research Open Access option.

This article, published in Genome Research, is available under a Creative Commons License (Attribution 4.0 International), as described at http://creativecommons.org/licenses/by/4.0/.

Receive free email alerts when new articles cite this article - sign up in the box at the top right corner of the article or click here.

\section{Affordable, Accurate Sequencing.}

